\begin{tabular}{|l|c|c|c|}
\hline ACTA CLASSICA & LII. & 2016. & \\
UNIV. SCIENT. DEBRECEN. & & & pp. 39-51. \\
\hline
\end{tabular}

\title{
DIE HAUT DES SATYRS: MARSYAS UND APOLLO
}

\author{
VON ÁGNES DARAB
}

\begin{abstract}
The essay proceeds from the observation that out of the surviving literature of antiquity only one poet, Ovid pays significant attention to the tragic fate of Marsyas. Both the Fasti and the Metamorphoses relate the tale. The narrative in Metamorphoses only focuses on the naturalistic description of the punishment, the flaying of Marsyas. The interpretation of this account within even wider contexts leads to the proposition that Marsyas's tale is the self-reflection of the elegiac poet Ovid, and as such it becomes a key narrative within Metamorphoses.

Keywords: hybris in the Metamorphoses, artist in Augustan literature, Apollo and the poets, narratology.
\end{abstract}

Dante invoziert in den einleitenden Versen der Beschreibung des Paradieses ${ }^{1}$ Apollo (buono Apollo), damit der antike Gott der Dichtung ihm helfe, den letzten großen Gesang der Divina Commedia und damit das ganze Werk zu vollenden. Dabei lässt er die Geschichte der Gottheit und des Marsyas in völlig neuer und einzigartiger Interpretation erscheinen. Damit die Krönung der Arbeit gelinge (a l'ultimo lavoro), bittet er Apollo, in seine Haut zu schlüpfen (entra nel petto mio) und ihm seine göttlich-musische Kraft zu verleihen (e spira tue), wie er es einst mit Marsyas getan habe (sì come quando Marsïa traesti de la vagina de le membra sue). Die Erwähnung des Marsyas in diesem Kontext ist für sich allein schon überraschend. Die feine Allusion auf die des Körpers beraubte Haut aber ist eine derart euphemistische Formulierung der Häutung des Satyrs, dass damit der antike Mythos eigentlich umgeschrieben wird. Unter den großen Büßern der antiken Mythologie gibt es nämlich kaum einen zweiten, der eine so grausame Strafe erleiden musste, und zwar durch Apollo, wie der phrygische Satyr.

Die ältesten verbalen und visuellen Narrative des Marsyas-Mythos tauchen im 5. Jahrhundert v. Chr. auf. ${ }^{2}$ Als seltene Ausnahme erscheint seine Darstellung ohne jede Vorgeschichte in der griechischen Kultur, und zwar gleichzeitig

\footnotetext{
${ }^{1}$ Dante Alighieri, Divina Commedia: Paradiso 1, 13-21.

${ }^{2}$ Visser 1999, 955.

doi 10.22315/ACD/2016/3
} 
in der Literatur und in der bildenden Kunst. ${ }^{3}$ Die Beschreibungen von Herodot, ${ }^{4}$ Xenophon ${ }^{5}$ und Platon ${ }^{6}$ halten schon fast alle Komponenten der kanonischen Form des Mythos fest: dass Marsyas phrygisch und ein Satyr war, sein virtuoses aủ入ós-Spiel, sein Wettkampf mit Apollo und dessen tragischer Ausgang, seine Häutung. Die griechischen Quellen aus dem 5-4. Jahrhundert legen den Schwerpunkt aber nicht auf die úßpıotńs-Natur des Satyrs, sondern einerseits

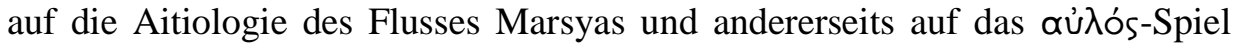

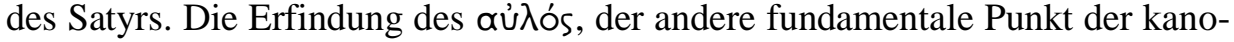
nischen Form des Mythos, fehlt in allen drei Beschreibungen. Im phrygischen

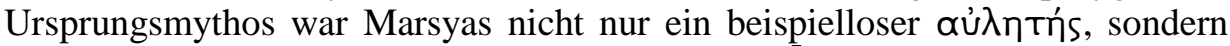
offensichtlich auch der Erfinder des Instruments. ${ }^{7}$ Diesen Ursprung bewahrt auch noch mehrere Jahrhunderte später der Erfinderkatalog im 7. Buch von Plinius dem Älteren: „Musicam Amphion, fistulam et monaulum Pan Mercuri, obliquam tibiam Midas in Phrygia, geminas tibias Marsyas in eadem gente, Lydios modulos Amphion, Dorios Thamyras Thrax, Phrygios Marsyas Phryx." 8

Das Auftauchen von Athena als Erfinderin, das dann zum Ausgangspunkt des Sujets der kanonischen Geschichte wurde, ist eine griechische Weiterentwicklung des Mythos. ${ }^{9}$ Die ältesten Schrift- und Bilddokumente, die eine derartige Transformation des Mythos zeigen, stammen ebenfalls aus dem 5. Jahrhundert. Das Dithyrambos-Fragment des Melanippides von Melos mit dem Titel Marsyas (440/439 v. Chr.) hält denselben Augenblick fest wie die erste bildliche Darstellung des Athena-Marsyas-Mythos, Myrons auf 450 datiertes Figurenensemble: ${ }^{10}$ das Fortschleudern der Pfeife. Das Auftauchen Athenas als Erfinderin stellt eine radikale Umformung des Mythos dar. Marsyas wurde vom

\footnotetext{
${ }^{3} \mathrm{Zu}$ den Schriftquellen s. Burckhardt 1930, in letzter Zeit und ausführlicher Volk 1995. Für die Darstellungen in der bildenden Kunst: Weis 1992.

${ }^{4}$ Hdt., VII 26.

${ }^{5}$ Xen., An. I $2,8$.

${ }^{6}$ Plat., Symp. 215b-d

7 Über Marsyas als Erfinder des Aulos und des Kybele-Gesangs s. Weis 1992, 366; Volk 1995, 13; Visser 1999, 955.

${ }^{8}$ Plin., Nat. VII 204: Die Musik erfand Amphion, die Rohr- und die einfache Hirtenflöte Pan, der Sohn des Hermes, die Querflöte Midas in Phrygien, die Doppelflöte Marsyas im gleichen Volke; die lydische Tonart lehrte Amphion, die dorische der Thraker Thamyras, die phrygische der Phryger Marsyas, die Kithara Amphion, nach anderen Orpheus und Linos. Übersetzung zitiert aus: C. Plinius Secundus d. Ä.: Naturkunde, Buch VII. Anthropologie. Herausgeben und übersetzt von Roderich König in Zusammenarbeit mit Gerhard Winkler. Artemis \& Winkler Verlag, Zürich / Düsseldorf, 1996. (Tusculum)

${ }^{9}$ Burckhardt 1930, 1989; Volk 1995, 13.

${ }^{10}$ Weis 1992, 376. Das Fortschleudern des Aulos findet sich auch in einem zeitgenössischen Dramenfragment (TGF Nauck fr. 381): Volk 1995, 14.
} 
Erfinder des aủ入ós zum bloßen Finder des Instruments degradiert, ${ }^{11}$ womit sich die Geschichte in die hellenisierte Aitiologie des aủ入ós verwandelte. ${ }^{12}$

In der hellenistischen Kunst verschob sich der Schwerpunkt der verbalen und visuellen Narrative gleichermaßen in Richtung Wettstreit und vor allem Bestrafung. Die Hauptstimme der Elegien des Archias von Mytilene ${ }^{13}$ und des Alkaios von Lesbos ${ }^{14}$ ist die Trauer. Das zentrale Bild des Gedichts des Archias ist der an einer Fichte aufgehängte Leib des Satyrs, das des Gedichts von Alkaios der an den Baum gefesselte Marsyas. Diese beiden auf das 2-1. Jahrhundert v. Chr. datierten Dichtungen sind Dokumente einer wichtigen Veränderung, die auch bei den bildlichen Darstellungen des Mythos bereits in klassischer Zeit zu beobachten ist. Das Hauptthema der Darstellungen des 4. Jahr-

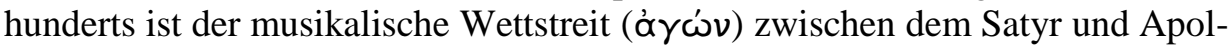
lo, in der zweiten Hälfte des Jahrhunderts tauchen aber auch zwei ikonografische Typen der Vorbereitung der Bestrafung auf: der mit hinter dem Rücken gefesselten Händen hockende (religatus) und der an einen Baum gefesselte (adligatus) Marsyas. ${ }^{15}$ Die perspektivisch bedeutendste Neuerung der bildenden Kunst des Hellenismus ist aber die Schaffung des dritten, hanging Marsyas genannten, ikonografischen Typs, von dem die späteren Darstellungen des Themas grundlegend bestimmt werden. ${ }^{16}$

Bis zum 1. Jahrhundert v. Chr. erhält also der Mythos sein kanonisches

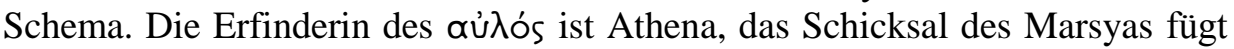
sich endgültig in die Reihe der vielen üßpıs-Geschichten ein. Zugleich widmet Ovid als einziger in der römischen Literatur dem tragischen Schicksals des Satyrs seine Aufmerksamkeit, und zwar eine ganz besondere: sowohl die Fasti, ${ }^{17}$ als

\footnotetext{
${ }^{11}$ Zum Vorgang der Verschmelzung der beiden Geschichten s. Junker 2002, 158-159.

${ }^{12}$ Die Umformung des Mythos wird üblicherweise im Kontext des Diskurses über die zeitgenössische neue Musik interpretiert: Schauenburg 1958, 58-59; Weis 1992, 367; Volk 1995, 14; Visser 1999, 955. Klaus Junker plädiert für eine Neuinterpretation, wonach Athena den Aulos nicht wegwirft, sondern ihn dem Marsyas übergibt, damit der Satyr die Sterblichen gleichsam als Kulturvermittler mit dieser Musik bekannt mache: Junker 2002, 145-158.

${ }^{13}$ Anth. Pal. VII 696.

${ }^{14}$ Anth. Pal. XVI 8.

${ }^{15}$ Weis 1992, 373; Salvo 2008, 92-95.

${ }^{16}$ Weis 1992, 374 und 377; Salvo 2008, 93-94. Anne Weis hat diesem ikonografischen Typ eine ganze Monografie gewidmet. Sie unterscheidet zwei Typen des hanging Marsyas (red und white); erstere datiert sie auf den späten Hellenismus, letzteren auf die Mitte des 1. Jahrhunderts n. Chr.: Weis 1992/a. Ihr folgt Salvo 2008, 93-95.

${ }^{17}$ Ov., Fast. VI 695-710.
} 
auch die Metamorphoses ${ }^{18}$ erzählen die Geschichte, in völlig unterschiedlichen Narrativen. ${ }^{19}$

Protagonistin und Erzählerin des Passus aus den Fasti ist Minerva, die als tibia inventrix die Geschichte heraufbeschwört: wie sie das Instrument erfunden habe (697-698: Prima terebrato per rara foramina buxo, / ut daret effeci, tibia longa sonos), warum sie ihre Erfindung weggeworfen habe (700: vidi virgineas intumuisse genas), welche ein namentlich nicht genannter Satyr gefunden habe (703: Inventam satyrus). Die Fortsetzung (703-709), die beinahe die Hälfte der fünfzehn Verse umfassenden Passage ausmacht, erzählt dennoch die Geschichte des anonymen Satyrs. Jede der sieben Verse baut auf je einem Verb auf, aus deren Kette sich die Tragödie des Marsyas herauskristallisiert. Der von (inventam) miratur - (sonum) sensit - (auras) dimittit et concipit gebildete Vorgang ist das Glück der Entdeckung und des Spiels. Die Wende konzentriert sich im superbus erat, hier schlägt die Erzählung in eine Hybris-Geschichte um, deren tragischer Ausgang unvermeidbar ist: provocat (et Phoebum) - (superante) pependit - (membra a cute) recesserunt.

Auch die persönliche Geschichte Minervas hat ein dramatisches Moment: die Erkenntnis, dass ihre Erfindung ihre göttliche Schönheit verzerrt: „Ars mihi non tanti est; valeas, mea tibia!" dixi. ${ }^{20}$ Dieser Satz allein baut einen Dialog zwischen den Marsyas-Episoden in den Fasti und den Metamorphoses auf. In letzteren schreit Marsyas während seiner Häutung: ,a! piget, a! non est” clamabat „tibia tanti” ${ }^{21}$ Zitierte Stelle alludiert dabei intertextuell auf das dritte Buch der Ars amatoria, wo Minervas Fall als Beispiel dafür fungiert, dass der Groll das Gesicht verzerrt: ,, i procul hinc” dixit ", non es mihi, tibia, tanti”, / ut vidit vultus Pallas in amne. ${ }^{22}$ In Ovids Ars von Minerva und in den Metamorphosen von Marsyas gesprochene Satz konstituiert nicht nur den Dialog der drei Werke, ${ }^{23}$ sondern schafft auch den Kontext, in dem die Geschichte inter-

\footnotetext{
${ }^{18}$ Ov., Met. VI 382-400.

${ }^{19}$ Andrew Feldherr und Paula James sehen in der Zerstückelung der Erzählung die narrative Abbildung der Häutung des Marsyas: Feldherr - James 2004, 78.

${ }^{20}$ Ov., Fast. VI 701.

${ }^{21}$ Ov., Met. VI 386

22 Ov., Ars III 505-506.

${ }^{23}$ Franz Bömer betont an den ovidschen Anwendungen des Satzes einerseits die dichterische Virtuosität, andererseits auf der Variation von tibia / ars basiert seine Datierung der Szene in den Metamorphoses auf eine späteren Zeitpunkt als die in den Fasti: Bömer 1976, 109-110. Andrew Feldherr sieht - sich auf Alessandro Barchiesi berufend - in der Intertextualität der Aussage der Minerva und im mit dixi angezeigten Selbstzitat die Möglichkeit, das non est tanti als Ovids auf sich selbst bezogene Aussage zu lesen. Feldherr entwickelt diese Interpretation dahingehend weiter, das der Satz dadurch besondere Bedeutung erhalte, dass es sich bei dem 6. Gesang der Fasti um eine Dichtung nach der Verbannung handle, während Ars diejenige Dichtung sei, die für seine relegatio verantwortlich gewesen sei: Feldherr - James 2004, 78.
} 
pretiert werden muss: was ist das virtuose künstlerische Vermögen wert, was im weiteren Sinn die Kunst, wo sind die Grenzen des Künstlers und der Kunst? Der Intertext baut den Raum der Interpretation auf, in den das Marsyas-Narrativ der Metamorphoses eingebettet ist. ${ }^{24}$

Der Mythos fügt sich als eingebettete Erzählung in die Textur der Metamorphoses. Als sie von den Folgen der Hybris der Niobe hören, ${ }^{25}$ erinnern sich die lykischen Bauern an zwei frühere Fälle der Rache von Latona und ihren Kindern. So wird zunächst der Ursprungsmythos der Frösche ${ }^{26}$ und dann die Geschichte des Marsyas ${ }^{27}$ vorgebracht, eine in mehrfacher Hinsicht in Verlegenheit bringende Erzählung. Den Rahmen der kurzen Erzählung bilden der nur ein paar Worte umfassende Verweis auf den Sieg Apollos (384-385: Quem Tritoniaca Latous harundine victum, / Adfecit poena.) und das Moment der Verwandlung am Schluss. Die Erzählung wird aber nicht mit der Metamorphose des Marsyas abgeschlossen, sondern mit der Trauer seiner Waldgefährten, mit der Verwandlung ihrer Tränen. Zugleich wird der aus den Tränen der Naturwesen entstehende Fluss - als ein Stück Natur - als ewiges Monument seiner Existenz dennoch zum Denkmal des Marsyas. ${ }^{28}$

In Verlegenheit bringend ist diese Erzählung zudem, weil es sich bei dem objektiv erzählten Sieg Apollos sowie bei der von Trauer und Verwandlung gerahmten "Geschichte” in Wahrheit um eine brutale Hinrichtung handelt: die Häutung des Satyrs mit einer einzigen Handbewegung ${ }^{29}$ und dann die naturalistische Beschreibung des zur lebendigen Wunde gemachten Leibes ${ }^{30}$. Eine derart schaudervolle Szene findet man weder in der antiken Literatur, noch in den Metamorphoses. Nach dem ästhetischen Urteil von Lessing sei die Beschreibung zwar abstoßend, aber im Textzusammenhang dennoch angemessen. ${ }^{31}$ Franz Bömer ordnet sie der Kategorie des schwarzen Humors zu, ${ }^{32}$ Karl Galinsky beurteilt die in den Metamorphoses vorkommenden sadistischen und perversen Geschichten im Allgemeinen dahingehend, dass Ovid Gefallen an grotesker Grausamkeit gefunden habe und außerdem dem zeitgenössischen Geschmack entsprechen habe wollen, welcher durch die in den Arenen zu se-

\footnotetext{
${ }^{24}$ Zur narrativen Technik der einander interpretierenden Intertextualität der ovidschen (und anderer) Texte s. am ausführlichsten: Barchiesi 2001, 49ff.

${ }^{25}$ Ov., Met. VI 146-312.

${ }^{26}$ Ov., Met. VI 313-381.

${ }^{27}$ Ov., Met. VI 382-400.

${ }^{28}$ Zur Metamorphose als Thema und Gattung bei Ovid und zu ihren narrativen Merkmalen und zahlreichen Interpretationsmöglichkeiten s. Feldherr 2002.

${ }^{29}$ Ov., Met. VI 387: Clamanti cutis est summos direpta per artus.

${ }^{30}$ Ov., Met. VI 388: Nec quiqam nisi vulnus erat.

${ }^{31}$ Lessing 1968, 183-184.

${ }^{32}$ Bömer 1976, 108.
} 
henden Gemetzel und die pantomimisch vorgetragenen, schreckenerregenden, sensationslüsternen Mythos-Szenen bestimmt wurde. ${ }^{33}$ Nach Eleanor W. Leach sei bei dieser Beschreibung von mehr die Rede als von „Ovids gewöhnlichem Horror”, Marsyas gehäuteter Leib sei eine Metapher des seiner künstlerischen Ambitionen Beraubten. ${ }^{34}$ Die Grundlegung der Interpretation von Elena Theodorakopoulos bildet das corpus in metaphorischem Sinn: sowohl Körper, als auch Text. Die Häutung bedeutet also das Berauben der Integrität des schaffendes self und der Schaffung. ${ }^{35}$ In der ästhetisch-poetologischen Interpretation von Charles Segal stellt Ovid mit dem Bild des lebendigen Leibes die Frage, wodurch das Abstoßende und das Schöne hervorgerufen würden, wo die Grenze zwischen den beiden ästhetischen Qualitäten zu ziehen sei. ${ }^{36}$ Philip Hardie leitet alle gewaltsam induzierten Verwandlungs-Narrative der Metamorphoses aus dem im Prolog exponierten Thema ab (In nova fert animus mutatas dicere formas / corpora): demzufolge könne die Verwandlung der Körper nicht nur von übernatürlichen Erscheinungen, sondern auch von Gewalttaten verursacht werden ${ }^{37}$ Nach Andrew Feldherrs origineller Analyse beruhe die Beschreibung auf einem Wortspiel. Die noch zuckenden Sehnen, die pulsierenden Adern von Marsyas' gehäutetem Leib seien zugleich Attribute Apollos. Der Glanz der Sonne (perlucentes, micant), die Sehne des Bogens (nervi) und die Saiten der Lyra (nervi, fibras) erinnerten auch nach dem Tod des Satyrs noch an Apollos Sieg. ${ }^{38}$ Aber wenn wir im schnellen Strömen des zum Fluss gewordenen Marsyas auch den virtuosen Lauf des Spiels der tibia hören, ${ }^{39}$ dann setzt der zuckende Leib doch nicht dem Sieg des Apollo ein Denkmal. In der Metamorphose können wir dann nicht die Unsterblichkeit des Marsyas, sondern die der mit ihm eins gewordenen Musik, die der von ihm vertretenen Kunst sehen.

\footnotetext{
${ }^{33}$ Galinsky 1975, 138-143. Die Wirkung der spectacula und venationes auf das Narrativ der Metamorphoses schließen auch Feldherr und James nicht aus, sie formulieren aber viel vorsichtiger als Galinsky: Feldherr - James 2004, 86-87.

${ }^{34}$ Leach $1974,127$.

35 Theodorakopoulos 1999, 154-155.

${ }^{36}$ Segal 1998, 34.

${ }^{37}$ Hardie 2002, 40-41.

${ }^{38}$ Feldherr - James 2004, 82-83. In seiner Monographie interpretiert Andrew Feldherr die Szene in weiterer Zusammenhang (Feldherr 2010, 105): „If we take Marsyas as a sympathetic figure, whose status as an artist we are prepared to endorse, then Ovid's potential distance from this figure not only lessens the political implications involved in taking on the voice of Apollo's rival but more importantly reveals that even an autonomous artist is caught up in a larger context that inevitably exerts his pressure over the meaning of his words and, indeed, determines the very modes and language available to him."

${ }^{39}$ Ov., Met. VI 399: Inde petens rapidum ripis declivibus aequor.
} 
Nach weiteren Quellen waren die Richter des musikalischen Wettstreites entweder die Bewohner von Nysa, ${ }^{40}$ oder die Musen, ${ }^{41}$ oder der Berg Tmolos, ${ }^{42}$ Apollos Sieg aber war nicht mit leuteren Mitteln errungen: er machte es zur Bedingung, dass die Wettstreitenden ihre Instrumente auch verkehrt herum spielen, ${ }^{43}$ bzw. dabei auch singen. ${ }^{44}$ Nichts davon erwähnt Ovid. Dagegen führt er Apollo unter dem Namen Latous in die Erzählung ein und verweist damit auf die vorangehenden zwei Geschichten, auf die Rache der Latona und ihrer Kinder, zurück. ${ }^{45}$ Das lässt keinen Zweifel daran offen, dass der Rachefeldzug weitergeht. Eine Rekapitulation des Vorausgegangenen ist deshalb nicht nötig: Marsyas erscheint in den Metamorphoses nicht als auletes und nicht als hybristes, sondern gleich als victus.

In Ovids Erzählung ist Apollo nicht zu sehen und nicht zu hören. Die Bedeutung davon zeigt sich in ihrem ganzen Ausmaß im Kontrast, der im zweiten dichterischen certamen, der Erzählung vom Wettstreit zwischen Apollo und Pan, entsteht. ${ }^{46}$ Im Zentrum der Erzählung steht hier der Wettkampf selbst, in dem Apollo so erscheint, wie wir uns nach der Beschreibung des Properz ${ }^{47}$ die Kultstatue im Apollo-Tempel auf dem Palatin vorstellen können, der kitharodos Apollo: mit einem Lorbeerkranz auf seinen goldblonden Locken, einem bis zum Boden reichenden, purpurnen Mantel und einer Lyra in Händen. Hier wird der idealisierte Apollo der augusteischen Zeit sichtbar, die Marsyas-Erzählung zeigt davon aber nichts. Die Musikalität des Wettstreits mit Pan ${ }^{48}$ wird durch Stille, sein Glanz ${ }^{49}$ vom durch den gehäuteten Leib durchschimmernden Licht, der Purpur von Apollos Mantel ${ }^{50}$ durch Marsyas' Blut abgelöst.

Es ist ein Gemeinplatz der Fachliteratur, dass in der augusteischen Zeit jede künstlerische Darstellung in Verbindung mit Apollo eine politische Stellungnahme war. Apollo war damals nicht nur der Gott der Dichtung, sondern eine zu politischen Zwecken herangezogene Gottheit, ${ }^{51}$ das göttliche alter ego ${ }^{52}$ des

\footnotetext{
${ }^{40}$ Diod. III 58.

${ }^{41}$ Paus. VIII 9, 1; Lukian, Dial. deor. XVI 2.

${ }^{42}$ Myth. Vat. I 90

${ }^{43}$ Apollod. I 4, 2.

${ }^{44}$ Hyg., Fab. 165; Diod. III 59.

${ }^{45}$ Eine Analyse der Passion der Kinder bzw. des Narrativs unter Gender-Gesichtspunkten bietet Segal 1998, 26.

${ }^{46}$ Ov., Met. XI 146-179.

${ }^{47}$ Prop. II 31.

${ }^{48}$ Ov., Met. XI 163: carmine delenit; 170: dulcedine captus

${ }^{49}$ Ov., Met. XI 165: caput flavum; 167: instructamque fidem gemmis et dentibus Indis / sustinet a laeva

${ }^{50}$ Ov., Met. XI 166: Tyrio saturata murice palla.

${ }^{51}$ Galinsky 1996, 215-219.

${ }^{52}$ Galinsky 1996, 188: „his divine alter ego, Apollo”.
} 
Augustus, dem er nach Actium oder unmittelbar davor neben seinem Haus auf dem Palatin einen Tempel errichtete; in dem er denjenigen sah, der ihm im Kampf bei Actium zum Sieg verholfen habe und für den er nach dem Sieg und zur Erinnerung an diesen die Stadt Nikopolis gründete sowie ein Heiligtum und einen riesigen Altar zu Ehren des Apollo Actius errichtete. ${ }^{53}$ Ovid stellt Marsyas nicht den Apollo des Mythos, sondern den des Augustus gegenüber. Diesen Apollo lässt er nicht als kitharodos, sondern als Rächer auftreten. Wo doch die Gegenüberstellung des Marsyas mit dem Gott des Gesangs nicht nur durch das musikalische certamen motiviert wäre. Auch auf Grund des Apollo-Bildes der Literatur und der bildenden Kunst der augusteischen Zeit blicken wir mit Recht mit dieser Erwartung auf den Text. Insgesamt wurde Apollo in der visuellen Propaganda als Gott des Gesangs dargestellt, ${ }^{54}$ die Rolle des Dichters und die politische Propaganda wurden also nicht getrennt. In der Literatur der Zeit wird Apollo, ob als Phoebus Apollo oder als heilender Gott, mit kühler Erhabenheit, aber als den Dichtern helfende und sie inspirierende Gottheit dargestellt. ${ }^{55}$

In den Metamorphoses aber wird weder die unterstützende Rolle der Musen noch die des Apollo erwähnt. Apollo erscheint überhaupt nur in entehrenden Situationen oder wütet. Das Bild des Daphne hinterherlaufenden Gottes ist nur der erste Schritt seiner Entthronung in den Metamorphoses. Der Wandteppich der Arachne zeigt nämlich unter den caelestia crimina (VI 131) nur die erotischen Abenteuer des Gottes, ${ }^{56}$ was zu der strukturell-inhaltlichen Einheit der Metamorphoses führt, welche den Kontext für die meiner Meinung nach wichtigste und tiefste Bedeutungsschicht des Marsyas-Narrativs bildet.

Mit dem certamen von Arachne und Minerva beginnen nicht nur die Wettstreit-, und damit die hybris-Geschichten, ${ }^{57}$ sondern auch die Künstlergeschichten. In den Metamorphoses finden sich sämtliche mythischen Künstlerfiguren

\footnotetext{
${ }^{53}$ Zanker 1987, 90-96; Hoff - Stroh - Zimmermann 2014, 90-95. Die neueste monografische Aufarbeitung des ganzen Fragenkomplexes liefert Miller 2009.

54 Von der Innendekoration des Apollo-Tempels auf dem Palatin und der Apollo als kitharodos darstellenden Statue darin gibt besonders das Gedicht von Properz ein plastisches Bild: II 31. Zur Erscheinung der augusteischen Zeit, der Indienstnahme griechischer Schöpfungen der bildenden Kunst für die neue Machtideologie s. Pollitt 1978, besonders 164-167. Die beste und vollständigste Untersuchung zum Thema ist bis heute Zanker 1987.

${ }^{55}$ Die Verwendung von Literatur für politische Zwecke zeigt am Beispiel der Entstehungsgeschichte der Aeneas-Sage Gruen 1993. Zur Politisierung der Literatur der augusteischen Zeit s. Galinsky 1996, 225-244, neuerdings Hoff - Stroh - Zimmermann 2014, 145-157, am differenziertesten Miller 2009, 298-331.

${ }^{56}$ Ov., Met. VI 122-124.

57 Zur künstlerischen varietas, wie Ovid das 5. und das 6. Buch mit Hilfe der Person Minervas sowie dem Wettstreit der Mnemoniden und Pieriden, also mit Hilfe des Wettstreitmotivs, verbindet und dieses dann mit Hilfe weiterer Figuren und Anspielungen ins 7. Buch weiterführt, s. Bömer 1976, 11.
} 
in der mittleren Pentade (Bücher VI-XI). ${ }^{58}$ Schon diese Positionierung hebt die Künstlergeschichten aus dem Ganzen des Textes hervor. Arachne und Marsyas finden sich im VI. Buch, Dädalus ${ }^{59}$ im VIII., Orpheus und Pygmalion im X., gefolgt vom Zerreißen des Orpheus im XI. ${ }^{60}$ und gleich darauf Pans Wettstreit, welcher als Geschichte in sich unabgeschlossen bleibt und nur als Begründung für die Eselsohren des Königs Midas dient. Sterbliche können die Götter nicht besiegen. Dennoch wagen die Künstler der Metamorphoses, die eher selbstbewusst als hybristes sind, den Wettstreit. Ovid macht klar, dass sie dies nicht ohne Grund tun. An Arachnes Wandteppich finden selbst Minerva und der Neid nichts auszusetzen. ${ }^{61}$ Marsyas Musik wird, wie wir gesehen haben, in keiner einzigen Erzählung schlechter beurteilt als das Lyra-Spiel Apollos. Dädalus und Orpheus erreichen in Wahrheit das Unmögliche. Sie erobern den Himmel und die Unterwelt und damit den für die Sterblichen nicht zugänglichen Raum.

In den Metamorphoses taucht Apollo als Gott der Musik und der Dichtung insgesamt zwölfmal auf, ${ }^{62}$ davon achtmal in der mittleren Pentade. Er ist aber nicht nur als kitharodos - gerade in den Künstlergeschichten der Metamorphoses am grausamsten. Wir haben - in diesem Kontext - allen Grund, in der Sonne, die das Wachs zum Schmelzen bringt, das die Flügel von Ikarus zusammenhält, Phoebus zu sehen. Wie auch dazu, in der Bezeichnung vates für Orpheus ${ }^{63}$ eine Allusion auf den Gott Apollo zu erblicken. Niobe ist keine Künstlerin, aber die Frau des neben Orpheus berühmtesten Lyra-Spielers und

\footnotetext{
${ }^{58}$ Die Forschungen zur Struktur der Metamorphoses plädieren zum Teil für eine Zweiteilung, zum Teil für eine zyklische Struktur, die meisten aber favorisieren eine Dreiteilung in je fünf Bücher. Letztere begründen manche mit den Figuren der Bücher: Götter - Heroen - geschichtliche Personen, andere mit den zentralen Schauplätzen: Theben - Athen - Troja und Rom, oder mit den Abschlüssen der aus je fünf Büchern bestehenden Einheiten: die Erzählungen über die Muse - Orpheus - Pythagoras. Robert Colemans Studie zeigt auf, dass Ovid die chronologische Basis des Geschichtenerzählens ( $a$ b origine mundi ad mea ... tempora) mit zahlreichen weiteren textstrukturierenden Gesichtspunkten anreichert, ohne dabei die Linearität des ganzen Werks aufzubrechen: Coleman 1971. In unserem Zusammenhang ist wichtig, dass die Dreiteilung nur von Michael von Albrecht auf „musische Grundlage” gestellt wird: Albrecht 1994, 636: „Nur in diesen Büchern ist von den Musen die Rede.” und 640: „Die Musen erscheinen später, und zwar - gewiß nicht zufällig - in den Schlußbüchern der drei Pentaden.”

${ }^{59}$ Zur Interpretation im Kontext der Künstlergeschichten s. Ritoók 1995.

${ }^{60}$ Eleanor W. Leach interpretiert die Geschichten von Arachne, Pygmalion und Orpheus in den Metamorphoses im vielschichtigen Kontext des Künstlers und seines Schaffens: Leach 1974, 102-126.

${ }^{61}$ Ov., Met. VI 129-130: Non illud Pallas, non illud carpere Livor / possit opus.

${ }^{62}$ Fontenrose 1940, 432: Met. I 518, 559; II 601, 682 f; VI 384 (Marsyas); VIII 15f; X 108 (Gesang des Orpheus), 170, 205; XI 155 (Pan), 165-171 (Pan), 317.

${ }^{63}$ Ov., Met. XI 2, 8.
} 
Sängers, des völlig unschuldigen Amphion, der in Folge der Tragödie Selbstmord begeht. ${ }^{64}$

Persönlich und als handelnde Figur und nicht nur als Anspielung oder metaphorisch erscheint Apollo in den Künstlergeschichten nur in der Geschichte von Marsyas. Unter den Künstlern in den Metamorphoses erleidet Marsyas die schrecklichste Strafe und in dieser Geschichte zeigt Apollo sein dunkelstes Gesicht. So wie wir im Kontext der mittleren Pentade der Metamorphoses von Apollo mit Recht denken, dass er nicht als der Gott des Mythos straft, sondern als Repräsentant der zum neuen goldenen Zeitalter erklärten Welt des Divus Augustus,${ }^{65}$ können wir im Büßer, in Marsyas ebenfalls mit Recht die mythische Verkörperung des Meisters der mit der tibia begleiteten Gattung, der Elegie, sehen. Kein Satyr, sondern der Elegiendichter Marsyas erleidet hier den schrecklichen Foltertod und wir irren uns wohl kaum, wenn wir in dieser unverhältnismäßigen Bestrafung die Selbstreflexion des römischen Meisters der Elegie, des unter der Bedrohung der Verbannung lebenden Ovids, sehen. ${ }^{66}$ Unter den Künstlergeschichten gibt es nur eine, die nicht tragisch endet: die vom Bildhauer Pygmalion, dessen Belohnung die umgekehrte Metamorphose ist. Gleichsam als Inversion der Marsyas-Geschichte sind der lebendige Leib und das Pulsieren der Adern nicht die letzten, sondern die ersten Zeichen des Lebens. Marsyas' lebendiger Leib wird zum Ende der Erzählung zur bloßen Wunde: vulnus erat. Pygmalions leblose Statue wird zum Leib: corpus erat. ${ }^{67}$ Die unverzichtbare göttliche Unterstützung bleibt nicht aus, diese Rolle wird aber von Venus erfüllt, die das erstrangige und in Ovids Fall einzige Thema der

\footnotetext{
${ }^{64}$ Ov., Met. VI 401-402.

${ }^{65}$ Wie Feldherr und James $(2004,85$.) es formulieren: „His Apollo cannot be read in isolation from Augustus'."

${ }^{66}$ Feldherr und James kommen, allerdings auf Grund einer anderen Logik, zu einer ähnlichen Schlussfolgerung. Sie sehen in der Erzählung eine verdoppelte Rolle Ovids: er sei der Narrator des Todes von Marsyas und auch Marsyas selbst. Den Hintergrund dazu beschreiben sie als die Situation der Auslieferung, in der sich der Dichter gegenüber Augustus befunden habe: Feldherr - James 2004, 84-85. Die Welt der Metamorphoses ist grundlegend dadurch gekennzeichnet, dass das Schicksal ihrer Figuren sich unerwartet von Grund auf ändert. Patricia Johnson interpretiert dies im Zusammenhang mit den wettstreitenden Künstlern als Ovids ,nachträgliche Prophezeiung" (hindsight): Johnson 2008, 121. Charles Segal nimmt mit dieser Eigenart im Hintergrund die Erfahrung wahr, die Ovid gemacht hat, die kaiserliche Anordnung seiner Verbannung: Segal 1998, 36. Letztere beiden Interpretationen sind selbstverständlich nicht trennbar von der unbeantwortbaren Frage der Datierung der Metamorphoses. Johnsons Interpretation stützt sich auf eine Datierung vor der Verbannung, Segals auf eine nach der relegatio. Ob wir sie nun als Erfahrung interpretieren oder - wie auch ich glaube - als Vorausahnung, auf jeden Fall können wir die plötzlichen Wendungen des Schicksals in den Künstlergeschichten als Metapher der völligen oder zumindest weitgehenden Einschränkung der künstlerischen Freiheit auffassen.

${ }^{67}$ Ov., Met. X 289: corpus erat! saliunt temptatae pollice venae.
} 
Elegiendichter, die Liebe, verkörpernde Göttin. Sie versteht, was Pygmalion nicht auszusprechen wagt, ${ }^{68}$ und verstünde auch, was Ovid zusammen mit Marsyas an Apollo-Augustus gerichtet schreit: die Kunst, die Elegiendichtung, ist nur Spiel - non est tibia tanti!

In der augusteischen Zeit verkündete die Kunst die Ewigkeit von Roms Macht. In diesem politisch-kulturellen Umfeld bedeutete das Verfassen eines Textes, dessen Hauptbotschaft die ständige Veränderung und Metamorphose der Welt ist, eine ebensolche provocatio wie diejenigen, denen die Künstler der Metamorphoses zum Opfer fielen. Das Leitmotiv von Vergils Aeneis ist das Schaffen: das Schaffen von Ordnung nach dem Chaos. ${ }^{69}$ Die Metamorphoses führen gerade Beispiele des Zerfalls vor und das meist mit Beispielen der Verwandlung des menschlichen Körpers, in Extremfällen dessen Zerreißens. Minerva zerreißt wütend Arachnes Wandteppich. Apollo reißt die Haut von Marsyas' Leib. Die thrakischen Bacchantinnen zerreißen Orpheus. Die ungezwungen und unterhaltsam scheinende Welt der Metamorphoses ist in Wahrheit eine Welt der Bedrohung, in der immer und überall Gewalt ausbrechen kann. ${ }^{70}$

Die Marsyas-Erzählung widerspricht nicht nur mit ihrem Inhalt, sondern auch mit ihrer Ästhetik spektakulär dem Klassizismus, den die pax Augusta mit ihrer Ideologie für adäquat hielt. Das Abstoßende, das Schreckliche als Thema und als ästhetische Qualität hebt die Tragödie des Marsyas unter den Künstlergeschichten der Metamorphoses hervor und macht sie zum Schlüsselnarrativ des ganzen Werks. Von Proteus, der das zentrale Thema der Metamorphoses, den Gestaltwechsel, den proteischen Charakter der ovidschen Welt vertritt, ist in eben dieser Pentade, ja im Zentrum des gesamten Textes, am Beginn des VIII. Gesangs die Rede..$^{71}$ Die Häutung des Marsyas, in der die göttliche Grausamkeit gegenüber dem Künstler zum Extrem gesteigert wird, findet sich an der zentralen Stelle im VI. Buch. ${ }^{72}$ Die unterschiedliche, aber in beiden Fällen spektakuläre Positionierung der zwei Geschichten fordert auf den Leser zur metapoetischen Lektüre, nach der Ovid in ihnen und durch sie auf sein eigenes Künstlerschicksal und sein eigenes Text, die Metamorphoses, reflektiert. Mit der Einführung der Figur des Proteus stellt er das Thema des Werks, die endlose Verwandlung der Welt, in den Mittelpunkt, mit der Figur des Marsyas den

\footnotetext{
${ }^{68}$ Ov., Met. X 275-276:" sit coniunx, opto," non ausus ,, eburnea virgo"” / dicere, Pygmalion, , similis mea” dixit, ,, eburnae."

${ }^{69}$ Galinsky 1996, 246.

${ }^{70}$ Segal 1998, 32 .

${ }^{71}$ Ov., Met. VIII 725-737. Barchiesi sieht die Formulierung des Themas der Metamorphoses in der Pythagoras-Rede, die sich an einer nicht weniger prominenten Stelle, nämlich als beinahe letzte Erzählung im XV. Buch findet: Barchiesi 2001, 69ff.

${ }^{72}$ Leach $1974,118$.
} 
Schöpfer der Metamorphoses, den auch als Epiker Elegien dichtenden Ovid, mit der durch Apollo erlittenen Tragödie die offizielle Verurteilung der ovidschen Elegiendichtung. Das bedeutungslose, als bloße Überleitung erscheinende Marsyas-Narrativ wird zum Paradigma der poetischen und politischen Beurteilung der Elegiendichtung, zum im Ganzen Bekenntnis Ovids, zur Inszenierung seiner künstlerisch-menschlichen Existenzanalyse.

Ovid hat aus dem Ursprungsmythos eines phrygischen Flusses und des aulos ein grundsätzlich anderes, neue künstlerische Möglichkeiten bietendes Narrativ geformt, das von literaturgeschichtlicher Bedeutung ist. Mit den Metamorphoses als Ganzes und darin besonders mit der Marsyas-Erzählung führte er den Körper als anthropologische und ideologische Konstruktion in den kulturellen Diskurs ein. In Ovids poetischer Welt ist der Körper durchlässig, ausgeliefert, verletzlich und kann jederzeit vernichtet werden. ${ }^{73}$ Die Gewalt gegen den Körper kommt immer aus den höchsten Sphären der Hierarchie, von den allmächtigen Göttern. Vom zeitgenössischen Kontext aus können wir in all dem die immer stärkere Kontrolle des augusteischen Regimes sehen, die Gefährdung der Autonomie des Individuums ${ }^{74}$ und die auf der Körper-Metapher beruhende Verwandlung dieser Erfahrung in Dichtung. Das ovidsche Narrativ hat zum ersten Mal die künstlerischen Möglichkeiten dieser Diskurse erkennen lassen und damit den Weg für die nicht enden wollende Rezeption des Marsyas-Themas bereitet.

\section{Bibliographie}

Albrecht 1994 = von Albrecht, M.: Geschichte der römischen Literatur, Bd. 1. München. Barchiesi 2001 = Barchiesi, A.: Speaking Volumes. Narrative and Intertext in Ovid and other Latin Poets, edited and translated M. Fox, S. Marchesi. London.

Bömer 1976 = Bömer, F.: P. Ovidius Naso: Metamorphosen, Buch VI-VII, Kommentar von Franz Böhmer. Heidelberg.

Burckhardt 1930 = Burckhardt, J.: Marsyas (6). In: Realencyclopädie der classischen Altertumswissenschaft, XIV. 2. Stuttgart, 1986-1992.

Coleman 1971 = Coleman, R.: Structure and Intention in the Metamorphoses. Classical Quarterly 21/1, 461-477.

Feldherr 2002 = Feldherr, A.: Metamorphosis in the Metamorphoses. In: Hardie, Ph. (ed.): The Cambridge Companion to Ovid. Cambridge, 163-179.

$-2010=$ Feldherr, A.: Playing Gods. Ovid's Metamorphoses and the Politics of Fiction. Princeton-Oxford.

\footnotetext{
${ }^{73}$ Segal 1998, 10.

${ }^{74}$ Leach 1974, 127; Segal 1998, 32; Theodorakopoulos 1999, 154-155.
} 
Feldherr - James 2004 = Feldherr, A. - James, P.: Making the Most of Marsyas. Arethusa 37, 75-103.

Fontenrose $1940=$ Fontenrose, J. E.: Apollo and the Sun-God in Ovid. American Journal of Philology 61, 429-444.

Galinsky 1975 = Galinsky, K.: Ovid's Metamorphoses. An Introduction to the Basic Aspects. Oxford.

- 1996 = Galinsky, K.: Augustan Culture. An Interpretive Introduction. Princeton.

Gruen 1993 = Gruen, E. S.: Cultural Fiction and Cultural Identity. Transactions of the American Philological Association 123, 1-14.

Hardie 2002 = Hardie, Ph.: Ovid and early imperial literature. In: Hardie, Ph. (ed.): The Cambridge Companion to Ovid. Cambridge, 34-45.

Hoff - Stroh - Zimmermann 2014 = Hoff, R. von den - Stroh, W. - Zimmermann, M.: Divus Augustus. Der erste Römischer Kaiser und seine Welt. München.

Johnson 2008 = Johnson, P. J.: Ovid before Exile. Art and Punishment in the Metamorphoses. Wisconsin

Junker 2002 = Junker, K.: Die Athena-Marsyas-Gruppe des Myron. In: Jahrbuch des Deutschen Archäologischen Instituts, Bd. 117. Berlin-New York, 127-184.

Leach 1974 = Leach, E. W.: Ekphrasis and the Theme of Artistic Failure in Ovid's Metamorphoses. Ramus 3, 102-142.

Lessing 1968 = Lessing, G. E.: Laokoon oder über die Grenzen der Malerei und Poesie, Kap. 25. In: Rilla, P. (Hrsg.): Gotthold Ephraim Lessing, Gesammelte Werken in zehn Bänden, Bd. 5. Berlin-Weimar.

Miller 2009 = Miller, J. F.: Apollo, Augustus and the Poets. Cambridge.

Pollitt $1978=$ Pollitt, J. J.: The Impact of Greek Art on Rome. Transactions of the American Philological Association 108, 155-174.

Ritoók 1995 = Ritoók, Zs.: Amphion und Icarus. Acta Antiqua Academiae Scientiarum Hungaricae 36, 87-99.

Salvo 2008 = Salvo, G.: Ovidio come specchio della cultura figurativa di età Augustea. Miti di Hybris punita: Marsia. Eidola 5, 83-111.

Schauenburg 1958 = Schauenburg, K.: Marsyas. Römische Mitteilungen 65, 42-66.

Segal 1998 = Segal, Ch.: Ovid's Metamorphic Bodies. Arion 5/3, 9-41.

Theodorakopoulos 1999 = Theodorakopoulos, E.: Closure and Transformation in Ovid's Metamorphoses. In: Hardie, $\mathrm{Ph}$ - Barchiesi, A. - Hinds, S. (eds.): Ovidian Transformations. Essays on the Metamorphoses and ist Reception. Cambridge, 142-161.

Visser 1999 = Visser, E.: Marsyas. In: Der Neue Pauly, Bd. 7, 955.

Volk 1995 = Volk, K.: Marsyas in der antiken Literatur. In: Baumstark, R. - Volk, P. (Hrsg.): Apoll schindet Marsyas. Über das Schreckliche in der Kunst. Adam Lenckhardts Elfenbeingruppe. München, 13-18.

Weis 1992 = Weis, A.: Marsyas I. In: Lexicon Iconographicum Mythologiae Classicae V. 1. Zürich-München, 366-378.

— 1992/a. = Weis, A.: The Hanging Marsyas and it's Copies: Roman Innovation in a Hellenistic Tradition. Rome.

Zanker 1987 = Zanker, P.: Augustus und die Macht der Bilder. München.

(ISSN $0418-453 X)$ 